\title{
Band Gap Calculations of Ternary $\operatorname{InN}_{0.03125} \mathrm{P}_{0.96875}$ Alloy
}

\author{
M. UstundaG*, B.G. Yalcin, S. Bagci and M. Aslan \\ Sakarya University, Department of Physics, Sakarya, Turkey
}

\begin{abstract}
In the current study, structural and electronic properties of ternary dilute nitride $\operatorname{InN}_{x} \mathrm{P}_{1-x}$ alloys have been investigated by using density functional theory. The equilibrium lattice parameter of studied material has been calculated in zinc-blende phase. $2 \times 2 \times 2$ supercell with 64 atoms has been used for calculations. The lattice parameter of $\operatorname{InN}_{0.03125} \mathrm{P}_{0.96875}$ alloy is found to be $5.852 \AA$. By means of the equilibrium lattice parameter, electronic band structure has been calculated for dilute $3.125 \%$ nitride composition. It is found that a ternary $\mathrm{InN}_{0.03125} \mathrm{P}_{0.96875}$ alloy is a direct band gap semiconductor with energy band gap of $1.198 \mathrm{eV}$.
\end{abstract}

DOI: 10.12693/APhysPolA.128.B-135

PACS: 71.55.Ak, 71.15.Mb, 03.75.Hh, 71.90.+q

\section{Introduction}

$\mathrm{InN}_{x} \mathrm{P}_{1-x}$ semiconductor alloys have recently received considerable attention due to their applications in electronic and optoelectronic device technology. Indium compounds are useful for many applications due to their extreme properties such as hardness, low ionicity and high thermal conductivity. In this study, we have investigated structural and electronic properties of $\operatorname{InN}_{x} \mathrm{P}_{1-x}$ alloy at $3.125 \% \mathrm{~N}$ concentration. With this concentration, according to the best of our knowledge no experimental or theoretical study related to $\operatorname{InN}_{x} \mathrm{P}_{1-x}$ alloy have appeared in the literature. This work is important because experimental studies usually are performed at low concentrations. Also we expect that considerable contribution will be made to literature by means of this work. To reach low concentration, we have created 64atom $2 \times 2 \times 2$ supercell of InP and replaced a single $\mathrm{P}$ with N. This corresponds to an InNP alloy with $3.125 \%$ of $\mathrm{N}$ concentration. Therefore, the effects of $\mathrm{N}$ addition $(3.125 \%)$ to InP on lattice parameter and bulk modulus is determined. Furthermore, the change in band gap energy and bowing parameter at studied concentration is also determined.

The paper is organized as follows. The computational details are presented in Sect. 2, the calculated structural and electronic results are described in Sect. 3 and we have concluded the paper in Sect. 4.

\section{Method of calculation}

First principles calculations for $\operatorname{InN}_{0.03125} \mathrm{P}_{0.96875}$ semiconductor alloy are performed using the full potential linearized augmented plane wave (FP-LAPW) [1] method as implemented in WIEN2k [2] code within the framework of density functional theory (DFT). The exchange and correlation effects are treated using generalized gradient approach (GGA) based on Perdew et al. [3].

*corresponding author; e-mail: mustundag@sakarya.edu.tr
To get the total energy convergence, we have expanded the Khon-Sham wave functions up to $R_{\mathrm{MT}} K_{\max }=6$ $\left(R_{\mathrm{MT}}\right.$ is the plane wave radii and $K_{\max }$ is the maximum reciprocal lattice vector). Partial waves used inside atomic spheres have been expanded up to $l_{\max }=10$. In charge density Fourier expansion, magnitude of the largest vector $G$ is equal to $12 \mathrm{Ry}^{1 / 2}\left(G_{\max }=12 \mathrm{Ry}^{1 / 2}\right)$. The cut-off energy for separating core from valence states is set to $-6 \mathrm{Ry}$. The standard special $k$-points technique of Monkhorst and Pack (MP) [4] is used for accurate Brillouin zone integrations. We have used $2 \times 2 \times 2 \mathrm{MP}$ meshes and 8 special $k$-points are taken in the irreducible wedge of the Brillouin zone. The $k$-meshes and basis sets are chosen to be good enough such that the obtained results are well converged.

\section{Results and discussions}

In order to get optimum lattice parameter we have made volume optimization process for $\operatorname{InN}_{0.03125} \mathrm{P}_{0.96875}$. In Fig. 1, as an example, we have shown energy versus volume graph for studied alloy depending on the Birch-Murnaghan equation of state [5].

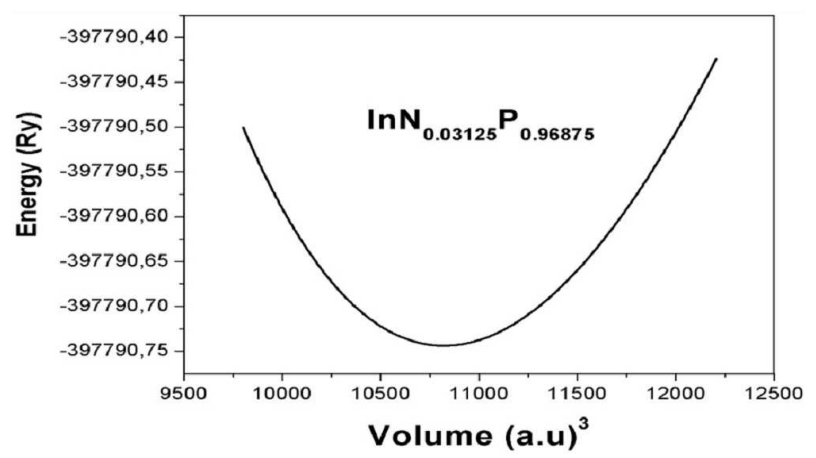

Fig. 1. Birch-Murnaghan equation of state for $\mathrm{InN}_{0.03125} \mathrm{P}_{0.96875}$ material.

The calculated lattice parameters and band gap energies of binary ( $\mathrm{InN}$ and $\mathrm{InP}$ ) compounds and ternary $\left(\mathrm{InN}_{0.03125} \mathrm{P}_{0.96875}\right)$ alloy are summarized in Table. 
The calculated lattice constant, bulk modulus and energy band gap values for binary compounds are in good agreement with other theoretical and experimental studies [6-14]. For ternary $\operatorname{InN}_{0.03125} \mathrm{P}_{0.96875}$ semiconductor alloy, as far as we are concerned, there is no theoretical or experimental study for structural and electronic properties for $3.125 \%$ concentration. It is important that the results are given in this study for the first time.

From Table, the lattice parameter of the calculated materials decreases with nitrate addition. The bulk modulus value $(B)$ which gives information about elasticity of material is represented in this table. As the same way, the $\mathrm{N}$ addition to $\mathrm{InP}$ increased the bulk modulus value that is compatible with the inverse proportion between bulk modulus and lattice parameter. We can clearly see from Table that replacing $\mathrm{P}$ with $\mathrm{N}$ in $\operatorname{InN}_{x} \mathrm{P}_{1-x}$ decreases band gap energy value for the studied material.

TABLE

Calculated lattice constant $(a)$, bulk modulus $(B)$, and band gap energies $\left(E_{\mathrm{g}}\right)$ for studied materials.

\begin{tabular}{c|c|c|c|c}
\hline \hline \multicolumn{2}{c|}{ Material } & $a[\AA]$ & $B[\mathrm{GPa}]$ & $E_{\mathrm{g}}[\mathrm{eV}]$ \\
\hline \multirow{3}{*}{$\mathrm{InP}$} & our result & 5.89 & 67.5 & 1.52 \\
& experiment & $5.86[6]$ & $71[6]$ & $1.424[7]$ \\
& theory & $5.94[8]$ & $68[8]$ & $1.5522[9]$ \\
\hline $\mathrm{InN}_{0.03125} \mathrm{P}_{0.96875}$ & our result & 5.85 & 68.02 & 1.19 \\
\hline \multirow{3}{*}{$\mathrm{InN}$} & our result & 5.00 & 139.1 & 0.65 \\
& experiment & $4.97[10]$ & $139[11]$ & $0.61[12]$ \\
& theory & $5.005[13]$ & $120.2[13]$ & $0.70[14]$
\end{tabular}

The calculated band gap values for $\mathrm{InN}$ and $\mathrm{InP}$ is represented in Fig. 2. Both $\mathrm{InN}$ and $\mathrm{InP}$ have direct band gap character. Therefore we expect that all concentrations of $\operatorname{InN}_{x} \mathrm{P}_{1-x}$ alloys also should have direct band gap character. As expected, we have found that the $\mathrm{InN}_{0.03125} \mathrm{P}_{0.96875}$ material has direct band gap with $1.19 \mathrm{eV}$ energy value as shown in Fig. 3.
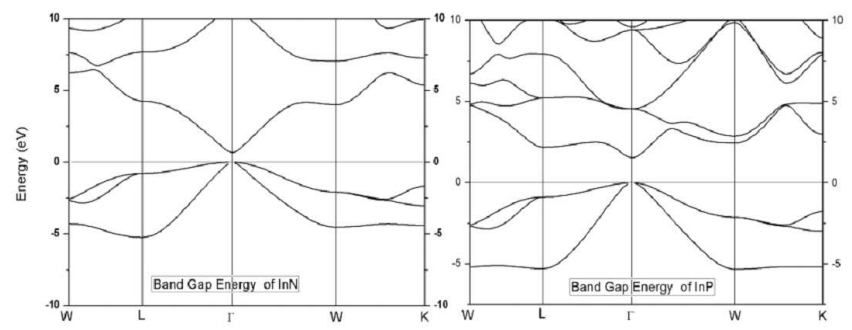

Fig. 2. Energy band gap diagram for $\mathrm{InN}$ and $\mathrm{InP}$ compounds.

In this study band gap energy values for InP and $\mathrm{InN}$ have been found as $1.52 \mathrm{eV}$ and $0.65 \mathrm{eV}$, respectively. The band gap value with $1.19 \mathrm{eV}$ for $\mathrm{InN}_{0.03125} \mathrm{P}_{0.96875}$ shows a nonlinear decrease which indicates existence of bowing.

In Figure 4, the relation between $\mathrm{N}$ concentration and band gap energy is represented. According to virtual crystal approximation [15] bowing parameter $(b)$ value

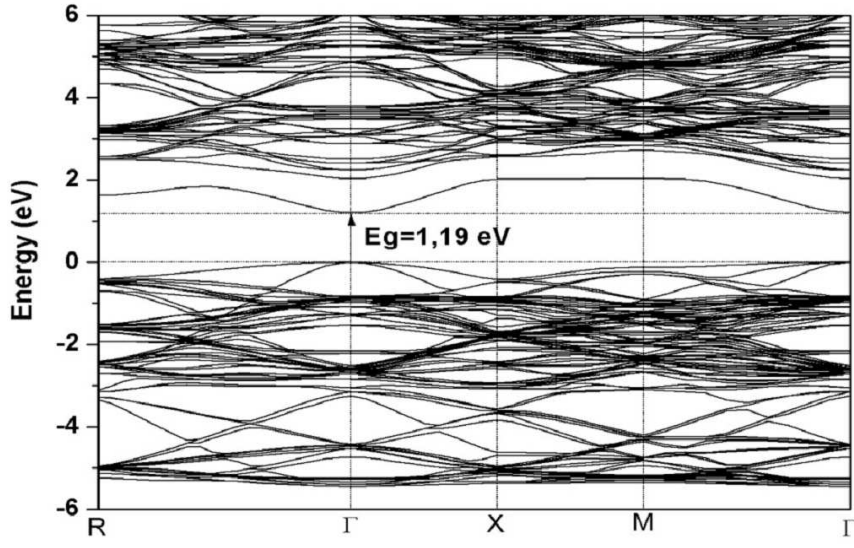

Fig. 3. Energy band gap diagram for $\mathrm{InN}_{0.03125} \mathrm{P}_{0.96875}$ alloy.

must be zero if there is no bowing. In this study by using nonlinear curve fitting, we have found 1.6 bowing parameter value at $3.125 \% \mathrm{~N}$ concentration. This indicates that the band gap bowing is inevitable for other $\mathrm{N}$ concentration of $\operatorname{InN}_{x} \mathrm{P}_{1-x}$ alloys.

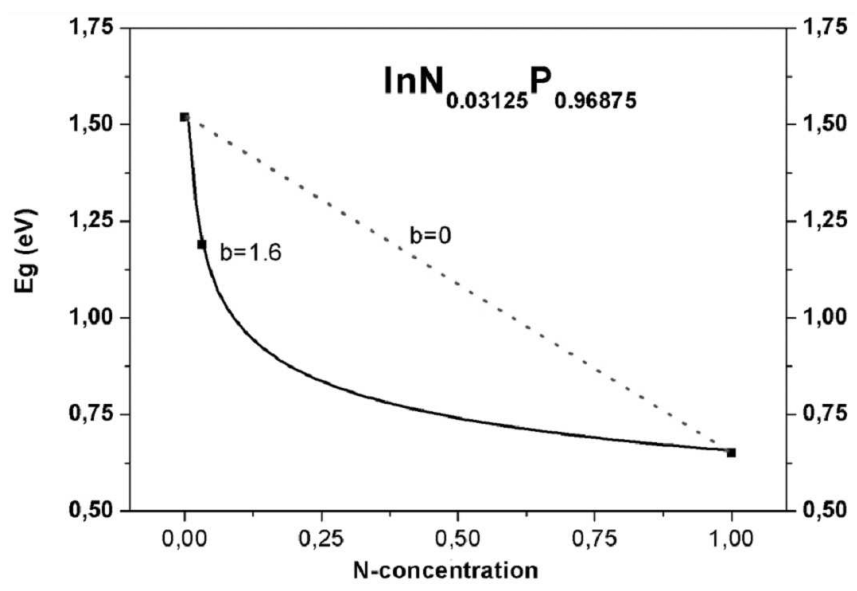

Fig. 4. The relation between $\mathrm{N}$ concentration and band gap energy for $\operatorname{InN}_{x} \mathrm{P}_{1-x}$ alloys.

\section{Conclusion}

We have studied the structural and electronic properties of ternary dilute nitride $\operatorname{InN}_{0.03125} \mathrm{P}_{0.96875}$ alloys by using density functional theory. The lattice parameter, bulk modulus and band gap values are calculated for studied materials. The effect of $\mathrm{N}$ addition to $\mathrm{InP}$ on these properties is determined. We have found that InN and InP have direct band gap and there is bowing at $3.125 \% \mathrm{~N}$ concentration. Therefore we expect that all concentrations of $\operatorname{InN}_{x} \mathrm{P}_{1-x}$ alloys have bowing parameter and could show direct band gap transition. We hope that this will light on future experimental and theoretical studies. 


\section{References}

[1] J.C. Slater, Adv. Quant. Chem. 1, 35 (1964).

[2] P. Blaha, K. Schwarz, G. Madsen, D. Kvasnicka, J. Luitz, An Augmented Plane Wave plus Local Orbital Program for Calculating the Crystal Properties, 12th ed., Wien 2012.

[3] J.P. Perdew, K. Burke, M. Ernzerhof, Phys. Rev. Lett. 7, 3865 (1996).

[4] H.J. Monkhorst, J.D. Pack, Phys. Rev. B 13, 5188 (1976).

[5] F. Birch, Phys. Rev. 71, 809 (1947).

[6] O. Madelung, L. Bornstein, Semiconductors, Physics of Group IV Elements and III-V, Compounds, Vol. 17, New Series, Group III, Springer Verlag, Berlin 1982.

[7] R.W.G. Wyckoff, Crystal Structures, 2nd ed., Krieger, Malabar 1986.

[8] A. Mujica, R.J. Needs, Phys. Rev. B 55, 9659 (1997).
[9] S.Z. Karazhanov, L.C. Lew Yan Voon, Semiconductors 39, 161 (2005).

[10] A. Tabata, A.P. Lima, L.K. Teles, L.M.R. Scolfaro, J.R. Leite, V. Lemos, B. Schöttker, T. Frey, D. Schikora, K. Lischka, Appl. Phys. Lett. 74, 362 (1999).

[11] A.U. Sheleg, V.A. Savastenko, Inorg. Mater. 15, 1598 (1979).

[12] J. Schörmann, D.J. As, K. Lischka, P. Schley, R. Goldhahn, S.F. Li, W. Löffler, M. Hetterich, H. Kalt, Appl. Phys. Lett. 89, 261903 (2006).

[13] M.E. Sherwin, T.J. Drummond, J. Appl. Phys. 69, 8423 (1991).

[14] S.Q. Wang, H.Q. Ye, J. Phys. Condens. Matter 14, 9579 (2002).

[15] J. Kim, M.V. Fischetti, J. Appl. Phys. 108, 013710 (2010). 